\title{
Ab initio Molecular Dynamics Study of Glycine Intramolecular Proton Transfer in Water
}

\author{
Kevin Leung and Susan B. Rempe \\ Sandia National Laboratories, MS $1415 \& 3$ 0310, Albuquerque, NM 87185
}

(Dated: September 10, 2018)

\begin{abstract}
We use $a b$ initio molecular dynamics simulations to quantify structural and thermodynamic properties of a model proton transfer reaction that converts a neutral glycine molecule, stable in the gas phase, to the zwitterion that predominates in aqueous solution. We compute the potential of mean force associated with the direct intramolecular proton transfer event in glycine. Structural analyses show that the average hydration number $\left(\mathrm{N}_{\mathrm{w}}\right)$ of glycine is not constant along the reaction coordinate, but rather progresses from $\mathrm{N}_{\mathrm{w}}=5$ in the neutral molecule to $\mathrm{N}_{\mathrm{w}}=8$ for the zwitterion. We report the free energy difference between the neutral and charged glycine molecules, and the free energy barrier to proton transfer. Finally, we identify approximations inherent in our method and estimate corresponding corrections to our reported thermodynamic predictions.
\end{abstract}

\section{INTRODUCTION}

Proton transfer plays diverse but fundamentally important roles in aqueous solution phase chemistry. Transfer reactions in water, aided by the indistinguishable nature of protons, produce highly mobile charge carriers utilized in fuel cells $\mathrm{s}^{\frac{1}{}}$ and biological systems ${ }^{2}$ for electrical energy conduction. Protonation and deprotonation reactions on ionizable substrates activate and modulate biochemical reactions. For example, in Rubisco, the enzyme that catalyzes the first reaction in carbon fixation, many steps in the reaction mechanism consist of protonation or deprotonation events on the enzyme-substrate complex ${ }^{3}$ In biological ion channels, changes in protonation states along the ion conduction path modify transport function,,$\frac{4}{4}$ and changes in protonation state in the gating region of some channels presumably trigger opening or closing of the channels $\frac{5}{\underline{5}}$ In order to understand such chemical processes associated with proton transfer in molecular detail, it is crucial to have methods that yield accurate estimates of the accompanying free energy changes.

Free energies, which characterize molecular interactions and govern the likelihood and rates of chemical reactions and conformational changes, are among the most important properties to be computed in aqueous environments. Since its introduction, the $a b$ initio molecular dynamics approach (AIMD) has demonstrated remarkable success in predicting and modeling the hydration structure, vibrational signature, and electronic properties of ions and molecules in water ${ }^{6}$ In recent years, it has also found increasing utility in computing thermodynamic properties, $\stackrel{7}{\rightarrow}$ including free energy differences by potential of mean force methods $\frac{8.9}{}$ These methods have successfully reproduced several pKa's for deprotonation reactions in water.

In fact, the recent introduction of various methods to calculate free energies, such as reaction-path finding techniques, ${ }^{10}$ targeted molecular dynamics ${ }^{11}$ and related methods, and a new theory of the liquid state that emphasizes the local hydration environment, 12 has created the potential for combining AIMD and unbiased, rigorous predictions of free energy barriers and differences. Relatively few aqueous phase free energy calculations exist in the literature based explicitly on AIMD data. To benchmark new advances in free energy methods, AIMD free energy calculations on simple, representative systems (especially proton transfer reactions) would be extremely valuable.

A convenient and popular model system for studying proton transfer events in aqueous environments is the intramolecular proton transfer of glycine in water. Water triggers zwitterion formation from the neutral molecule stable in the gas phase. Since the stability of the zwitterion relative to neutral conformers in water is entirely due to glycine-water interactions, 13 theoretical predictions for glycine in water are sensitive to the parameterization of water-glycine functional group interactions. This makes the glycine intramolecular proton transfer reaction a sensitive and valuable benchmark for calculating hydration effects on biological functional groups. In fact, when new methods are devised to sample molecular conformations at finite temperature quantum mechanically, glycine is often a model of choice as a first application. ${ }^{14.15}$

Part of the reason for the popularity of glycine as a model for proton transfer studies in proteins is that its tautomerization free energy difference and barriers have been measured 16.17.18.19.20.21.22 Glycine can exist in many neutral form conformers in addition to the zwitterion tautomer, as illustrated in Fig. 1. In the gas phase, the zwitterion (ZW) is unstable and spontaneously collapses to the neutral form (NF) conformer "IIp." 23.24 In contrast, the ZW is more stable than any neutral form in water due to large local charges and a large dipole moment. A free energy barrier of $14.3 \mathrm{kcal} / \mathrm{mol}$ is associated with the $\mathrm{ZW} \rightarrow \mathrm{NF}$ interconversion, ${ }^{20}$ and $\mathrm{ZW}$ is more stable by $7.27 \mathrm{kcal} / \mathrm{mol}^{17}$ From these results, the reverse, $\mathrm{NF} \rightarrow \mathrm{ZW}$ reaction is estimated to have a free energy barrier of $\sim 7 \mathrm{kcal} / \mathrm{mol}$ and there are indications 


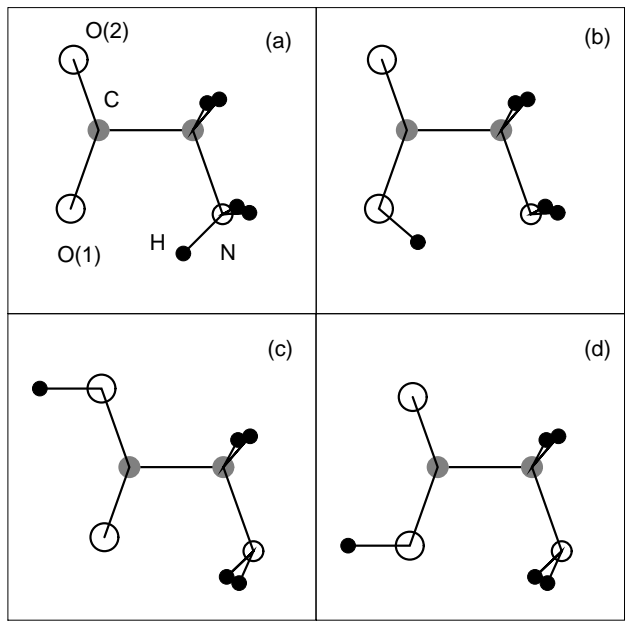

FIG. 1: Glycine tautomers. Black circles: H; grey: C; small and large open circles: $\mathrm{N}$ and $\mathrm{O}$, respectively. Panel (a): zwitterion (ZW) glycine; (b)-(d) three stable/metastable neutral form (NF) glycine molecules with configurations optimized in the gas phase, respectively, IIp, Ip, and IIIp. ${ }^{23}$ Note that some works in the literature label $\mathrm{O}(1)$ and $\mathrm{O}(2)$ in the reverse way.

that the $\mathrm{ZW} \rightarrow \mathrm{NF}$ free energy barrier is mainly entropic in origin 20 Other experimental data provides additional information on glycine hydration. Neutron scattering results on the hydration structure of the ammonium group in concentrated glycine solutions have been reported, ${ }^{22}$ as well as mass and size-selected photoelectron spectroscopic studies of hydrated glycine anions ${ }^{25}$

While experimental data makes glycine a choice system for study, comparison of theoretical and experimental studies of the proton transfer between the ZW and NF tautomers is complicated by the fact that the most stable aqueous NF conformer has not been identified ${ }^{26}$ The various NF conformers are related by rotation of the C - C, C - O, and C - N bonds. Conformer Ip (Fig. 1(c)) is the most stable structure in the gas phase while IIp is

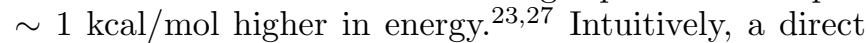
intramolecular proton transfer from ZW to NF in water should go first through IIp, which is closest in structure to ZW among the neutral form conformers. This is the proton transfer reaction that is most accessible to finite temperature, quantum mechanic (or quantum mechanics-based) simulations. Thus practitioners using a molecular dynamics simulation, based on a reactive force field fitted to Hartree-Fock data, ${ }^{28.29}$ have claimed to reproduce the experimental values for $\mathrm{ZW} \rightarrow \mathrm{NF}$ free energy difference and barrier by just considering the IIp neutral form. Other quantum chemistry works, however, find a small reverse IIp $\rightarrow \mathrm{ZW}$ barrier and interpret IIp as an intermediate, not the final or most stable NF product ${ }^{15.30 .31}$ To further complicate interpretation of the results, these quantum chemistry results depend on the details of the calculations and so are not necessarily in quantitative agreement with each other.

In this work, we do not seek the most favorable form of (metastable) NF glycine in aqueous solution. Instead, we use the ab initio molecular dynamics (AIMD) approach to focus on the direct IIp $\rightarrow \mathrm{ZW}$ proton transfer in water. AIMD treats the valence electrons of all atoms quantum mechanically, using density functional theory (DFT). It also samples glycine and water conformations at finite temperature via its molecular dynamics capability. While AIMD is computationally costly, it has several important advantages when treating intramolecular proton transfer in glycine. (a) Glycine itself is treated quantum mechanically, which is pertinent to the breaking and making of chemical (covalent) bonds that take place during proton transfer. (b) The number and conformations of water molecules in the first hydration shell, and the hydrogen bonds they form with the glycine atoms, are allowed to fluctuate and vary. This is significant because hydrogen bond network fluctuations have been known to be crucial in other prototypical proton transfer reactions in water ${ }^{32}$ (c) These first hydration shell water molecules are treated quantum mechanically. Widely used force field models for water and the carboxylate $\left(-\mathrm{COO}^{-}\right)$functional group, which is a crucial part of ZW glycine, predict carboxylate group hydration structures $33.34,35,36.37 .38$ that disagree with experiments 39.40 This is true even if the carboxylate group containing ion is treated quantum mechanically and only water is treated classically는 (i.e., a $\mathrm{QM} / \mathrm{MM}$ treatment ${ }^{42}$ ). In contrast, AIMD predictions of hydration structure are in good agreement with experiments $\underline{43}$ Likewise, in the one case where a QM/MM estimate of the ZW/IIp free energy difference is reported, the theoretical prediction overestimates the experimental value by a factor of four ${ }^{44}$ This emphasizes the sensitivity of this free energy difference to glycine-water interaction parameters.

The role of water conformational changes and waterglycine hydrogen bond fluctuations on the glycine intramolecular proton transfer reaction is central to this work. We dynamically sample water conformations using AIMD. These water configurational changes may be especially important for the $\mathrm{ZW} \rightarrow \mathrm{IIp}$ reaction. This is because the glycine dipole moment decreases along the reaction coordinate, leading to a reduction in the mean hydration number. Our work will reveal trends concerning hydration number variations and hydrogen bond network fluctuations as the intramolecular proton transfer proceeds.

A number of pioneering quantum chemistry works on glycine tautomerization have used a quantum treatment of the glycine molecule as well as a few water molecules in the glycine first hydration shell (i.e., a "supermolecule" approach). A static optimization of the supermolecule 
geometry is performed, and a polarizable dielectric continuum is used to treat the outlying water $\stackrel{15.31 .45}{=}$ The advantage of such methods is their relative computational ease, which allows the sampling of many glycine neutral form conformations.

In connection with these studies, our AIMD prediction of the progression in the average hydration number around each glycine atom, as the reaction coordinate varies, may contribute to future supermolecule studies of proton transfer reactions in water. Working within the framework of a statistical mechanical theory of liquids called quasi-chemical theory ${ }^{46}$ for example, accurate hydration free energies for metal ions have been computed by forming supermolecular clusters containing the metal ion and the full set of water molecules in the first hydration shell and embedded in a dielectric continuum model of bulk water 47.48 .49 .50 Clusters containing one glycine and 3 or 6 water molecules have been considered in recent published quantum chemistry studies $\frac{15.31 .45}{}$ As will be shown in this work, this is not sufficient to complete the first hydration shell of ZW glycine. We will make a preliminary attempt to quantify this effect.

The paper is organized as follows. Section 2 describes the method used. Our predicted potential of mean force and correlation functions are described in Sec. 3. Section 4 concludes the paper with further discussions. In the appendices, we discuss corrections to the AIMD results by estimating the effect of zero point energy contributions and of using different exchange-correlation functionals, and also we address the effect of using a finitesized simulation cell.

\section{METHOD}

We perform ab initio molecular dynamics simulations on a system with 1 glycine and $52 \mathrm{H}_{2} \mathrm{O}$ molecules. Finite size effects will be addressed in Appendix B using a simulation cell with $98 \mathrm{H}_{2} \mathrm{O}$ molecules. The Car-Parrinello Molecular Dynamics (CPMD) 51 code is applied, along with the BLYP gradient corrected exchange correlation functional ${ }^{52.53}$ and Troullier-Martins pseudopotentials ${ }^{54}$ BLYP has been shown to yield water-water pair correlation functions $g(r)^{7}$ as well as hydration structures of $\mathrm{NH}_{4}^{+}$and $\mathrm{HCOO}^{-}$ions that are in good agreement with experiments ${ }^{43.55}$ The effect of using other, perhaps more accurate, exchange correlation functionals will be addressed in Appendix A.

The simulation box is cubic with linear dimension $11.76 \AA$, which corresponds to a water density of 1.00 $\mathrm{g} / \mathrm{cm}^{3}$ plus the experimental glycine zwitterion volume of $72 \AA^{3}, \underline{56}$ The time step used is 5 a.u. (0.121 fs), and the deuterium mass is assumed for all protons throughout, although we will continue to use the word "proton." The temperature is kept at $\mathrm{T}=300 \mathrm{~K}$ using a thermostat unless otherwise stated.
We compute the potential of mean force, $\Delta G(R), 57$ along a reaction coordinate defined as the difference between the $\mathrm{N}-\mathrm{H}$ and $\mathrm{O}(1)-\mathrm{H}$ distance:

$$
R=R_{\mathrm{N}-\mathrm{H}}-R_{\mathrm{O}(1)-\mathrm{H}} .
$$

$\mathrm{O}(1)$ is the IIp glycine oxygen atom covalently bonded to the acidic proton, and $\mathrm{H}$ is the proton being directly transferred between $\mathrm{O}(1)$ and the nitrogen atom. Progression along this coordinate represents a direct proton transfer from the ZW to the IIp form of glycine. For the IIp neutral conformer (Fig. 1b), $R_{\mathrm{O}(1)-\mathrm{H}} \sim 1 \AA$, and $\mathrm{N}$ and $\mathrm{H}$ are not covalently bonded, making $R_{\mathrm{N}-\mathrm{H}}$ larger than $1.4 \AA$, and hence $R>0$. For the ZW glycine, $R_{\mathrm{N}-\mathrm{H}} \sim 1 \AA, \mathrm{O}(1)-\mathrm{H}$ is not covalently bonded, and $R<0$. We use the umbrella sampling method ${ }^{58}$ (with harmonic constraints or biasing potentials) to compute the free energy profile along this reaction coordinate. Since one of the sampling windows is the unconstrained glycine zwitterion, this allows us to examine the structure of water around stable, equilibrium zwitterion glycine, and compute the pertinent water-glycine pair correlation functions $(g(r))$ as well 59

The barrier height $\Delta G^{*}$ depends on the reaction pathway. A more sophisticated approach would be to use the path-sampling method ${ }^{60}$ which in principle can sample the possible reaction pathways weighted by the proper statistical mechanical weight. This is computationally expensive, however, and seldom implemented within an AIMD setting. For the relatively simple system of glycine intramolecular proton transfer, we expect our relatively simple reaction coordinate to be adequate. This is because the reaction coordinate is similar to the one considered in Ref. 31 - it describes a straightforward direct proton transfer event. Using a simple $\mathrm{N}-\mathrm{H}$ bond distance reaction coordinate, Kassab et al. obtain results similar to their own gas-phase transition state finding calculations ${ }^{31}$ Furthermore, when we initiate a glycine IIp conformation in water, we observe that it undergoes a direct, intramolecular proton transfer to the ZW form in less than 1 ps. This observation corroborates the reaction coordinate we have chosen.

While the two oxygen atoms in $\mathrm{ZW}$ are in principle equivalent, they interconvert on a relatively long time scale, and they can be treated as distinct for the duration of our AIMD trajectories. In contrast, we do observe rotation around the $\mathrm{N}-\mathrm{C}$ bond in our simulations, which is also known to occur in picosecond timescales ${ }^{61}$ The three protons bonded to the nitrogen atom in the ZW glycine are therefore equivalent in our simulations, and, at any time step, the relevant proton in Eq. 1 is taken as the one closest to $\mathrm{O}(1), 59$ To compute the free energy profile along $R$, a harmonic penalty function $V(R)=A_{i}\left(R-R_{i}\right)^{2}$ is used to constrain $R$ in six umbrella sampling windows with a progression of target distances $R_{i}$, where $A_{i}$ ranges from $0.7 \mathrm{eV}$ to $1.2 \mathrm{eV} / \mathrm{AA}$ (see Table【for details). Window (a) corresponds to ZW glycine, 


\begin{tabular}{||c|c|c|c|c|c|c||}
\hline window & $\mathrm{a}(\mathrm{ZW})$ & $\mathrm{b}$ & $\mathrm{c}$ & $\mathrm{d}$ & $\mathrm{e}$ & $\mathrm{f}(\mathrm{IIp})$ \\
\hline$R_{i}(\AA)$ & 0.0 & -0.8 & -0.5 & -0.1 & 0.2 & 0.7 \\
$A_{i}(\mathrm{eV})$ & 0.0 & 1.0 & 1.0 & 1.2 & 1.0 & 0.7 \\
\hline
\end{tabular}

TABLE I: Constraint parameters $A$ and $B$ for the umbrella sampling windows. See Eq. 1

which is stable and requires no constraint.

AIMD trajectories are initiated by inserting a $\mathrm{ZW}$ or IIp glycine molecule into a simulation box with 54 water molecules, which has been equilibrated previously using the empirical SPC force field for water ${ }^{62}$ Two water molecules overlapping the glycine molecule are removed. We first conduct a $10 \mathrm{ps} \mathrm{QM} / \mathrm{MM}$ molecular dynamics trajectory for ZW and IIp glycine ${ }^{63}$ The resulting molecular configurations are used as the starting points for the AIMD trajectory in the ZW and IIp windows (i.e., windows (a) and (f)). Using AIMD, the QM/MM IIp configuration is equilibrated for $4 \mathrm{ps}$, and then statistics are collected for the next 10 ps. The window (f) constraint (Table I) is always applied to keep the IIp glycine from undergoing intramolecular proton transfer. The starting configuration of window (e) is taken 8 ps into the IIp trajectory, equilibrated at the new constraint (Table I) for $6 \mathrm{ps}$, and run for another $10 \mathrm{ps}$. Window (d) is spawned from window (e) 5 ps into its trajectory, then equilibrated for 2 ps with the new constraint. As will be discussed, in windows (d) and (e), statistics are collected for 20 ps.

As for window (a), we first use AIMD to re-equilibrate a QM/MM ZW configuration at $\mathrm{T}=300 \mathrm{~K}$ for $10 \mathrm{ps}$. The final configuration of this trajectory is once again re-equilibrated at $\mathrm{T}=350 \mathrm{~K}$ for $1 \mathrm{ps}$, and then statistics are collected at this temperature for $10 \mathrm{ps.} \mathrm{Both} \mathrm{win-}$ dows (b) and (c) are spawned from the configuration at the end of the window (a) trajectory, equilibrated for 1 ps with the new constraint, and then statistics are collected for $10 \mathrm{ps}$. These two windows are thermostated at $\mathrm{T}=350$ and $300 \mathrm{~K}$, respectively. The reason for using a higher temperature, and the small effect this has on the potential of mean force, will be discussed later on.

Our AIMD trajectories within each sampling window last 10 or 20 ps. Our total AIMD trajectory length is similar to that used to correctly reproduce experimental results for deprotonation of histidine residues $\underline{\underline{8}}$ Note that the reactive force field based glycine intramolecular proton transfer work of Ref. 28 uses trajectories only a few times longer than the one reported in our work. Furthermore, our use of longer trajectories in the windows that contribute most to $\Delta G$, and a higher temperature for the ZW window that only weakly contributes to $\Delta G$ (see below) further reduces statistical uncertainties. We estimate the cumulative statistical uncertainty of our predicted $\Delta G$ and $\Delta G^{*}$ between IIp and ZW glycine to be of order $\sim 1.4 \mathrm{kcal} / \mathrm{mol}$. ( $\Delta G^{*}$ for the reverse reaction, IIp $\rightarrow$ ZW, is much smaller.) As a result, one limitation

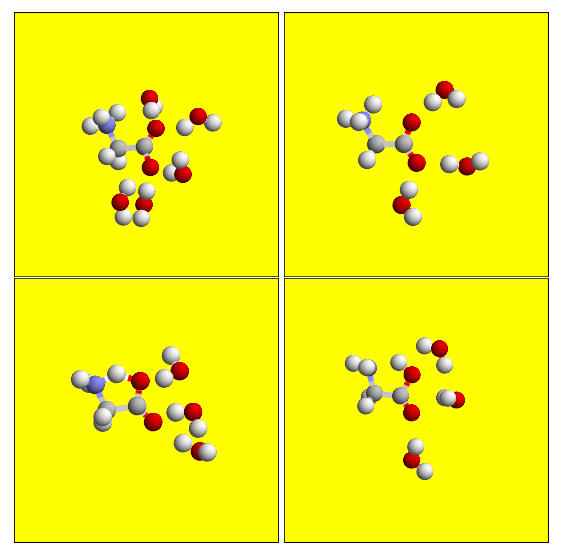

FIG. 2: Sample hydration structures around just the carboxylate/carboxylate acid group (not the amine group) in aqueous glycine. Only the first hydration shell is shown. Th grey, blue, red, and white spheres represent $\mathrm{C}, \mathrm{N}, \mathrm{O}$, and $\mathrm{H}$ atoms, respectively. Upper left panel: long lived zwitterion hydration shell configuration at $\mathrm{T}=300 \mathrm{~K}$. Upper right: zwitterion hydration shell after a trajectory of 5 ps at $\mathrm{T}=350 \mathrm{~K}$. Lower left: window (d) (transition state region); lower right: window (f) (neutral form glycine).

of our AIMD work is that we cannot resolve the temperature dependence of $\Delta G$ for this intramolecular proton transfer reaction. Nevertheless, the potential of mean force computed using AIMD potentially can be used to calibrate or refine force fields, which then can be applied to address the entropic contribution to $\Delta G$ accurately and efficiently.

\section{III. $A B$ INITIO MOLECULAR DYNAMICS RESULTS}

In this section, we first consider the hydration structures of the glycine functional groups. We also investigate the time dependence of the hydration numbers to demonstrate that the simulation time we use in each umbrella sampling window is adequate. Then we report the potential of mean force and hydration structures along the $\mathrm{ZW} \rightarrow \mathrm{IIp}$ reaction coordinate. Finally, we discuss our results in relation with experiments and quantum chemistry based supermolecule calculations.

\section{A. Zwitterion hydration structure}

ZW glycine, the most stable tautomer in water, has a large gas phase dipole moment and charge separation along the molecular framework that leads to strong interactions with water. Despite this, we expect, on the basis of work with formate ion hydration, ${ }^{43}$ that the co- 

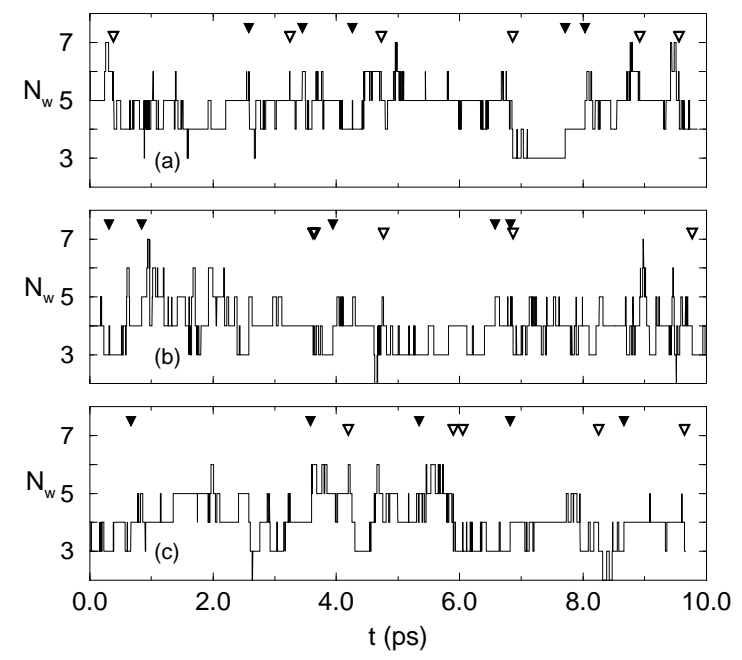

FIG. 3: Time dependent hydration numbers for $\mathrm{O}(1)$ and $\mathrm{O}(2)$ combined. (a) Window (a); (b) window (c); (c) window (d). Based on the first minimum of the glycine-water $g(r)$, we define a water molecule to be in the hydration shell if it one of its protons is within $2.5 \AA$ of the glycine oxygens. Filled and hollow triangles indicate where water molecules enter the hydration shell of $\mathrm{O}(1)$ or $\mathrm{O}(2)$ for the first time, or leave those hydration shells for at least 1 ps. There are numerous transient fluctuations and re-entrances into hydration shells. For concreteness, we arbitarily define a water molecule as "first" entering the hydration if it resides there for at least 1 ps later in the trajectory, and if it was not previously in the hydration shell for at least $2 \mathrm{ps}$.

ordination number of the carboxylate oxygens will experience large fluctuations $\underline{64}$ We indeed find that the combined instantaneous hydration number for these oxygens ranges from 3 to 7 , as illustrated in Fig. 3 . At $\mathrm{T}=300 \mathrm{~K}$, however, the first hydration shell of the $-\mathrm{COO}^{-}$group exhibits relatively slow dynamics during the first $\sim 10$ ps of the AIMD trajectory. For instance, in a 5 ps stretch, one of the water molecules bound to $\mathrm{O}(2)$ briefly leaves the hydration shell, is replaced by a second water, and then returns, displacing the newly added water molecule. This long-lived ZW configuration has two water molecules hydrogen bonded to $\mathrm{O}(1)$ and three to $\mathrm{O}(2)$ (see Fig. 2).

We did not observe similar slow dynamics in AIMD simulations of the aqueous formate ion ${ }^{43}$ using the BLYP exchange correlation functional, despite the fact that $\mathrm{HCOO}^{-}$is a charged species. We speculate that, whereas the carbon end of the formate ion is hydrophobic, the $-\mathrm{NH}^{3+}$ group in the $\mathrm{ZW}$ glycine forms another strong hydration shell and concentrates water molecules in the vicinity of the $\mathrm{COO}^{-}$group, causing a more persistent carboxylate-water interaction in the $\mathrm{ZW}$ glycine.
Regardless of the reason, while this long-lived hydration structure persists, the reaction coordinate $R$ will be locked into a relatively narrow distribution. As a result, sufficient statistics for the ZW glycine cannot be accumulated using a 10-20 ps trajectory. Instead, we collect statistics for windows (a) and (b) at $\mathrm{T}=350 \mathrm{~K}$. The dynamics at this higher temperature are much faster and involve several exchanges of water molecules between the first hydration shell and the bulk liquid. See Fig. 3.

As $R$ increases, the glycine dipole moment decreases, its interaction with water weakens, and the dynamics of water molecules around the glycine molecule become faster. Figure $3(\mathrm{~b})$ shows that the dynamics in window (c) and (d) are comparable to that in (a), despite the fact they are run at $\mathrm{T}=300 \mathrm{~K}$. This indicates that an elevated temperature is not needed for window (c) for a 10 ps trajectory. Figure 3 actually depicts two complementary quantities, the instantaneous hydration number of $\mathrm{O}(1)$ plus $\mathrm{O}(2)$, and the times at which the water molecules first enter or leave the hydration shells. The former includes all transient fluctuations and the rapid motion due to water molecules briefly forming and breaking hydrogen bonds with $\mathrm{O}(1)$ or $\mathrm{O}(2)$. The latter filters out the rapid fluctuations, but its definition is somewhat arbitary; we require that water molecules have at least a $1 \mathrm{ps}$ residence time to be so counted. At even higher temperatures, such that the time scale of exchange between first hydration shells and the bulk water region is faster than $1 \mathrm{ps}$, this criterion will have to be redefined.

We note that the residence times of water molecules in the hydration shells are sensitive to the strength of interaction between carboxylate oxygens and water molecules; a slight increase of just $1 \mathrm{kcal} / \mathrm{mol}$ will increase them significantly. Hence such quantities are expected to be sensitive to temperature as well as to the exchange correlation functional used $\underline{43}$

Figure 4 depicts the ZW glycine-water pair correlation functions, $g(r)$, for selected atomic sites. The two carboxylate oxygen atoms form an average of 4.7 total hydrogen bonds with water molecules. $\mathrm{O}(2)$ exhibits a hydration number $\mathrm{N}_{\mathrm{w}} \sim 2.66$, in good agreement with the hydration number of the formate ion computed using AIMD ${ }^{43} \mathrm{O}(1)$ forms an average of 2.0 hydrogen bonds with water molecules. It also forms an intramolecular hydrogen bond with one of the ammonium group protons $40 \%$ of the time, assuming a hydration shell radius of $2.5 \AA$. The combined hydration number of 4.7 is smaller than that of 5.3 predicted by Alagona et al ${ }^{35}$ using empirical force fields, and is much smaller than the hydration number of 7 for carboxylate groups predicted by Jorgensen and Gao's OPLS force fields 38 Integrating $g(r)$ between the ammonium protons and water oxygen atoms up to its first minimum, the ammonium group is found to have 3.0 water molecules in its first hydration shell. Using instead the $\mathrm{g}(\mathrm{r})$ between the nitrogen and water oxygen, we find $\mathrm{N}_{\mathrm{w}} \approx 4$ under its first peak, with 


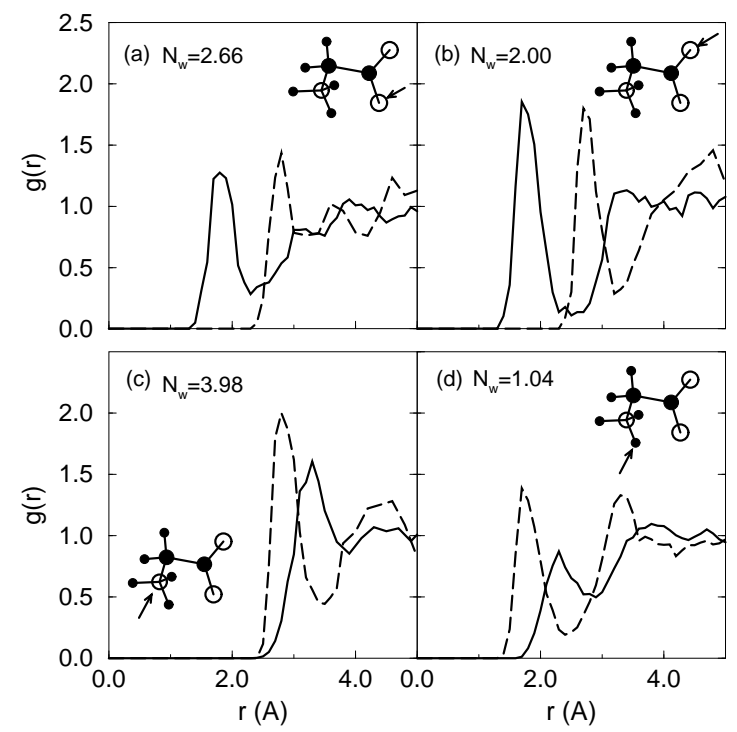

FIG. 4: Pair correlation functions between four atoms in ZW glycine, and oxygen (dashed line) and hydrogen atoms (solid line) on water molecules. (a) $\mathrm{O}(1)$ : (b) $\mathrm{O}(2)$ : (c) $\mathrm{N}$; (d) one of the three equivalent protons on the ammonium group. $\mathrm{N}_{w}$ indicates the number of water molecules around the atom, found by integrating to the first minimum in $\mathrm{g}(\mathrm{r})$ between the solute and water oxygen atoms.

an average $\mathrm{N}$ - $\mathrm{O}$ distance of $2.75 \AA$. A neutron scattering study for $5 \mathrm{~mol} \%$ glycine solution deduces a similar mean $\mathrm{N}-\mathrm{O}$ distance of $2.85 \pm 0.05$ and $N_{\mathrm{w}}=3.0 \pm 0.6$, based on analysis of the $\mathrm{N}-\mathrm{O}$ correlation ${ }^{22}$ Since the ammonium protons are directly hydrogen bonded to the water oxygens while the nitrogen atom is not, we deem the former more useful in determining the total number of hydrogen bonds, particularly because of the elevated temperature used in our simulations. Hence we estimate that AIMD predicts 3.0 hydrogen bonds between the ammonium group of ZW glycine and water molecules.

Unlike the carboxylate oxygens, the combined coordination number of the three protons in the $-\mathrm{NH}_{3}^{+}$do not exhibit large fluctuations, and thus these fluctuations should not contribute significantly to $\Delta G(R)$.

\section{B. Potential of mean force and hydration structure}

Figure 5 depicts $\Delta G(R)$ along the reaction coordinate $R$. The ZW glycine is found to be more stable than the IIp conformer by a free energy difference, $\Delta G$, of 11.2 $\mathrm{kcal} / \mathrm{mol}$. We also find a $12.7 \mathrm{kcal} / \mathrm{mol}$ transition state barrier, $\Delta G^{*}$, between these two forms of glycine located at $R \approx 0.2 \AA$. The statistical uncertainties in both $\Delta G$ and $\Delta G^{*}$ are estimated to be about $1.4 \mathrm{kcal} / \mathrm{mol}$. On the average, this transition state structure exhibits $\mathrm{O}(1)-\mathrm{H}$

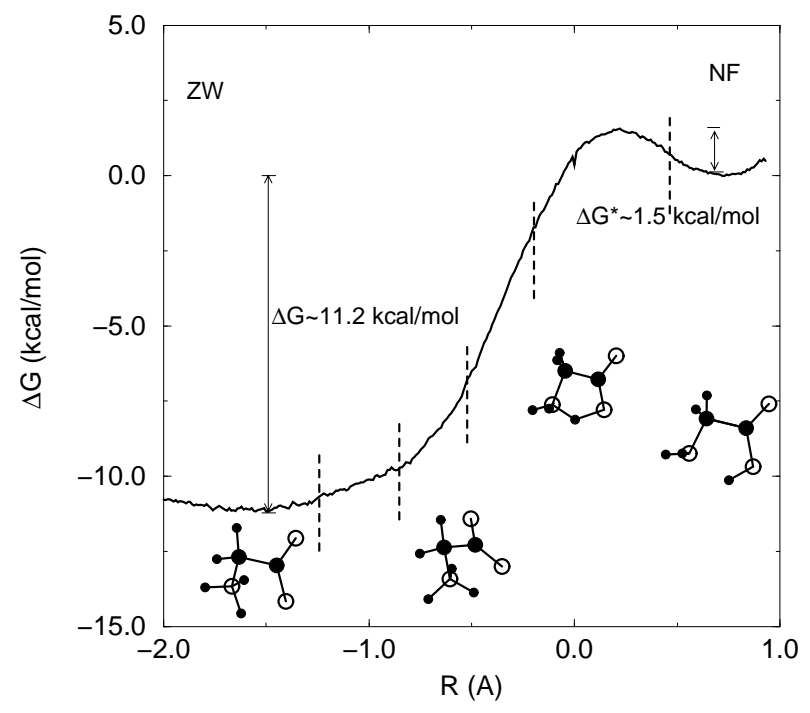

FIG. 5: Potential of mean force as a function of the chosen reaction coordinate $R=R_{\mathrm{N}-\mathrm{H}}-R_{\mathrm{O}-\mathrm{H}}$. The six umbrella sampling windows are demarcated with dashed lines, and snapshots of the glycine molecule in some of the windows are depicted.

and $\mathrm{N}$ - $\mathrm{H}$ distances of $\sim 1.1$ and $\sim 1.3 \AA$, respectively. We will consider several corrections to $\Delta G$ and $\Delta G^{*}$ below, but they will not qualitatively modify these conclusions.

Figure 6 plots as functions of $R$ the mean hydration numbers for $\mathrm{O}(1)$ plus $\mathrm{O}(2), \mathrm{N}$, and the proton being transfered. They are compiled across all six umbrella sampling windows. The results at the boundary of two windows are averaged. As $R$ increases, the ZW glycine continuously transforms to the IIp neutral form and the glycine dipole moment decreases, water becomes less structured around it, and the hydration number decreases for all glycine atoms we examined. In particular, when $R$ reflects a IIp neutral form configuration, the $\mathrm{NH}_{2}$ and $\mathrm{COOH}$ groups do not strongly bind to water molecules; each amine proton forms hydrogen bonds to only an average of $\sim 0.5$ water molecules. As a result, the hydration numbers drop almost by a factor of two in these cases.

As for the proton being transfered, it forms covalent and intramolecular hydrogen bonds with $\mathrm{N}$ and $\mathrm{O}(1)$ in the range $-0.8 \AA<R<0.7 \AA$, but forms no hydrogen bonds with water molecules. If $R$ is further increased, the intramolecular hydrogen bond cannot be sustained, and the proton begins to establish an intermolecular hydrogen bond with water molecules. Thus hydrogen bond network fluctuations are crucial to proton transfer, as observed in proton transport in water ${ }^{32}$ The statistical uncertainties in these $R$-dependent hydration numbers are of order $\sim 0.3$. Considering this, the hydration numbers are reasonably well converged, smooth functions of $R$. 


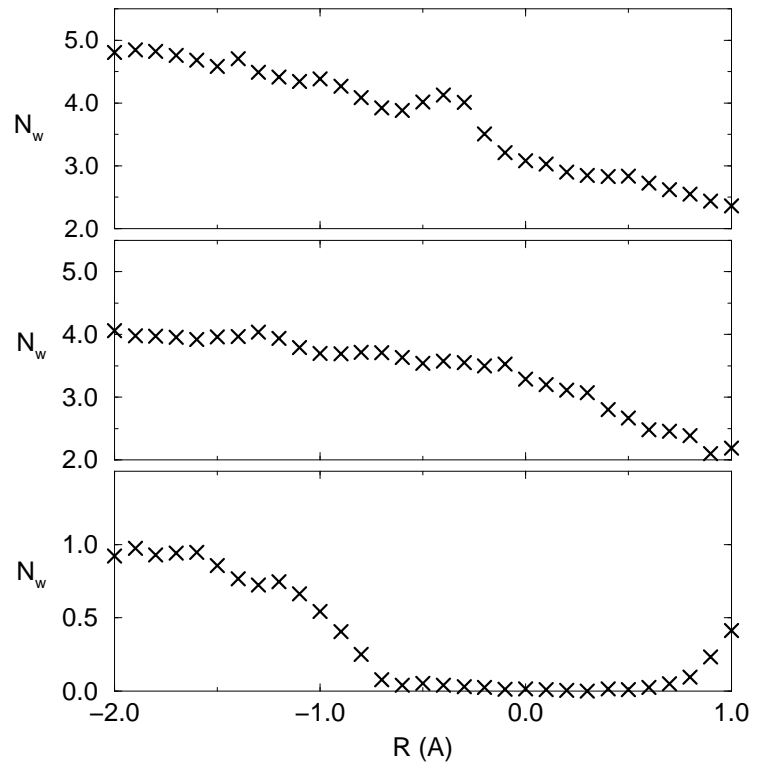

FIG. 6: $R$-dependent mean number of water molecules in the first hydration shell, $\mathrm{N}_{\mathrm{w}}$, for various atoms. (a) $\mathrm{O}(1)$ and $\mathrm{O}(2)$ combined; (b) $\mathrm{N}$; (c) $\mathrm{H}$ involved in intramolecular proton transfer. The cut-off criteria for determining these coordination numbers are: $\mathrm{O}-\mathrm{H}_{\mathrm{w}}$ distance of $2.5 \AA ; \mathrm{N}-\mathrm{O}_{\mathrm{w}}$ distance of $3.5 \AA$; and $\mathrm{H}$ - $\mathrm{O}_{\mathrm{w}}$ distance of $2.4 \AA$; respectively, where the subscript "w" refers to water molecules.

The predicted progression of hydration numbers should be valuable for future quasi-chemical calculations $\underline{46}$

At the transition state, where $R \sim 0.2 \AA$, the hydration numbers (Fig. 6) closely resemble those found for the IIp neutral form conformer, but differ significantly from that of the ZW.

To estimate the statistical uncertainties in our thermodynamic quantities, we define $\Delta \Delta G=\mid \Delta G\left(R_{2}\right)-$ $\Delta G\left(R_{1}\right) \mid$. For windows (b)-(e), $R_{2}\left(R_{1}\right)$ is the maximum (minimum) $R$ used in each window in Fig. 5. For windows (a) and (f), $R_{2}$ and $R_{1}$ are the $R$ values at the local minima, respectively. The statistical uncertainty in $\Delta \Delta G$ is estimated by dividing the 10 ps trajectory in each window into 4 blocks of 2.5 ps each. For windows (d) and (e), we run a trajectory twice as long. The estimated deviation from the mean computed using 2.5 and 5 ps blocks are almost identical for window (d), suggesting that the correlation time for $\Delta \Delta G$ is less than 2.5 ps. Recall that Fig. 3(c) shows only 5 instances each of "new" water molecules entering, and completely leaving, a hydration shell within the $10 \mathrm{ps}$ trajectory segment depicted in this window. There are also numerous transient fluctuations in $\mathrm{N}_{\mathrm{w}}$, however, and these fluctuations allow statistically meaningful sampling of $\Delta G(R)$. The correlation time appears slightly larger than 2.5 ps for window (e). Given that most windows are sampled for $10 \mathrm{ps}$, we report the cumulative uncertainty in $\Delta G$ as twice our estimated overall deviation from the mean estimated using 2.5 ps time blocks.

Recall also that windows (a) and (b) are sampled at $\mathrm{T}=350 \mathrm{~K}$. For these two windows, we estimate $\Delta G(R)$ at $\mathrm{T}=300 \mathrm{~K}$ by assuming that the free energy barrier is mainly due to entropy ${ }^{20}$ Then $\Delta G(R) /\left(k_{\mathrm{B}} T\right) \propto$ $\log P(R)$, where $P(R)$ is the probability, assumed to be temperature independent, that the reaction coordinate spontaneously exhibits value $R$. As shown in Fig. 5, $\Delta \Delta G$ in these two windows combined is $1.4 \mathrm{kcal} / \mathrm{mol}$. Even if $\Delta G(R)$ and $P(R)$ are entirely due to enthalpy, the resulting small error arising from sampling these windows at $\mathrm{T}=350 \mathrm{~K}$ will be a small fraction of $1.4 \mathrm{kcal} / \mathrm{mol}$, and will have little effect on the $\mathrm{ZW} \rightarrow \mathrm{IIp} \Delta G$.

\section{Neutral form glycines: spontaneous direct proton transfer}

A typical IIp neutral form glycine hydration structure is depicted in Fig. 2. The carboxyl oxygen and the two amine group protons of neutral form glycine exhibit hydration numbers of 2.1 and 1.0, respectively. These numbers are obtained using the constraint of Table \

In fact, we find that a constraint is necessary to stablize the IIp glycine molecule in AIMD and QM/MM trajectories. Upon releasing the constraint, the IIp molecule spontaneously undergoes a direct proton transfer to the ZW form in a sub-picosecond time scale, regardless of the starting configuration. This is consistent with the small proton transfer free energy barrier computed using umbrella sampling.

\section{Comparison with experiments}

We predict a $\mathrm{ZW} \rightarrow \mathrm{IIp}$ activation barrier $\left(\Delta G^{*}\right)$ of 12.7 $\mathrm{kcal} / \mathrm{mol}$, which is ostensibly within $1.6 \mathrm{kcal} / \mathrm{mol}$ of the reported experimental $\Delta G^{*}{ }^{20}$ We note that the experimental rate has been measured with both nuclear magnetic resonance $\frac{16}{}$ and the thermally modulated chemical relaxation method,, 20 and the reported intramolecular proton transfer rates are within $12 \%$ of each other. We also find a free energy difference for conversion from the ZW to the IIp conformer $(\Delta G)$ of $11.2 \mathrm{kcal} / \mathrm{mol}$, which is ostensibly $54 \%$ larger than the experimental value of $7.27 \mathrm{kcal} / \mathrm{mol},{ }^{17.65}$ As will be shown, various corrections to our AIMD free energies are small.

Thus we find, in qualitative agreement with quantum chemistry calculations $\stackrel{15.30}{\longleftarrow}$ that the experimental results do not correspond to the neutral form IIp conformer that is the focus of this study. The NMR result 16 states that the rate it measures is associated with a NF product that must undergo proton exchange with water before reverting back to the ZW form; the IIp conformer, which we predict to have a picosecond lifetime, will not qualify. 
It is likely that the observed rate ${ }^{16.20}$ involves the $\mathrm{ZW}$ first tranforming into the IIp molecule, and then on to one or several more stable NF conformers that undergo proton exchange in water and lead to the coalescence of NMR line shapes $\underline{16}$ The NMR free energy barrier will reflect a composite of these processes.

We have not computed the free energies and barriers of other NF conformers compared to the IIp neutral form. From our discussion in the previous section, our predicted $\Delta G=11.2 \mathrm{kcal} / \mathrm{mol}$ exceeds the experimental value of $\sim 7 \mathrm{kcal} / \mathrm{mol}$, which is consistent with the fact that IIp is only an intermediate, and a lower free energy conformer exists. The search for this stable conformer using AIMD will be left to future work.

We find that $\Delta G$ predicted by AIMD and the supermolecule approach, which uses one glycine and three water molecules plus dielectric continuum, differ by several $\mathrm{kcal} / \mathrm{mol}$ if BLYP is used in the latter case. This comparison will be presented in Appendix A along with the suggestion that the discrepancy exists because the supermolecule lacks a fully occupied first hydration shell.

\section{CONCLUSIONS}

Using ab initio molecular dynamics calculations and the BLYP exchange correlation functional, we find a $12.7 \mathrm{kcal} / \mathrm{mol}$ free energy barrier between zwitterion and conformer IIp of the neutral form glycine in water. The statistical uncertainty is estimated to be of order $1.4 \mathrm{kcal} / \mathrm{mol}$. We predict a $11.2 \pm 1.4 \mathrm{kcal} / \mathrm{mol}$ free energy difference between the zwitterion and this IIp conformer. The experimental free energy difference between the zwitterion and the neutral form is $7.27 \mathrm{kcal} / \mathrm{mol}$, although precisely which neutral form conformer dominates in water has not yet been determined experimentally, and will be addressed in future work.

We also gain useful qualitative insight on hydration structures from our AIMD simulations. We find that the hydration structure of the - $\mathrm{COO}^{-}$group in the zwitterion is similar to that of the formate ion, ${ }^{43}$ forming an average of 4.7 hydrogen bonds with water, while the $-\mathrm{NH}_{3}^{+}$group forms 3.0 hydrogen bonds with water molecules, yielding 8 water molecules in the nearest hydration shell of the ZW. The carboxyl oxygen and the two amine group protons of neutral form glycine exhibit hydration numbers of 2.1 and 1.0, respectively. The coordination numbers along the reaction coordinate interpolate between these limits.

This work demonstrates the viability of AIMD to predict free energy changes in aqueous reactions. AIMD allows fairly extensive sampling of the first hydration shell water configurations, which is found to have strong correlation with the progress along the reaction coordinate. While our conclusions about the crucial role of neutral form glycine conformers other than IIp is in agreement with some quantum chemistry supermolecule calculations (and in disagreement with reactive force field works 28.29 ), our quantitative results may be particularly valuable toward accurate parameterization of future quasi-chemistry calculations of proton transfer in aqueous environments. $\underline{\underline{46}}$

\section{ACKNOWLEDGEMENT}

We thank Todd Alam and Normand Modine for useful suggestions. This work was supported by the Department of Energy under Contract DE-AC04-94AL85000. Sandia is a multiprogram laboratory operated by Sandia Corporation, a Lockheed Martin Company, for the U.S. Department of Energy.

\section{Appendix A: Corrections to ab initio molecular dynamics results}

Several corrections to the thermodynamic quantities $\left(\Delta G\right.$ and $\left.\Delta G^{*}\right)$ determined from our AIMD trajectories should be considered. We show that they will not alter our conclusions. These include zero point energy corrections and use of a more sophisticated exchange correlation functional.

To estimate such corrections, we conduct gas phase cluster energy minimization calculations using the Gaussian code, with B3LYP 66 and BLYP exchange correlation functionals and various basis sets $\underline{67}$ Cluster optimizations are carried out in the $6-31 \mathrm{G}(\mathrm{d})$ basis, to reproduce results found in the literature, as well as in the $6-31+\mathrm{G}(\mathrm{d}, \mathrm{p})$ basis. Frequency calculations confirm that the predicted structures are true minima, and zero point energies are computed at the same level of theory. Refined single point estimates of energies are obtained using an extended $6-311+\mathrm{G}(2 \mathrm{~d}, \mathrm{p})$ basis set applied to the configurations found in the minimization calculations.

\section{A. Zero Point Energy}

The results in Sec. [IIB do not include zero point energy (ZPE) corrections. It is fairly costly to include ZPE effects in AIMD simulations via the path integral formalism. ZPE corrections, however, can be estimated during post processing of the trajectory data by appealing to supermolecule calculations. Here we consider a supermolecule geometry similar to that in Ref. 31. This cluster has one glycine and three water molecules, as shown in Fig. 7 , and a polarizable dielectric continuum mode ${ }^{68}$ is used to treat the bulk water boundary conditions. Using the basis and refined basis sets described above, we find that ZPE indeed raises the free energy of ZW glycine by only $\sim 1 \mathrm{kcal} / \mathrm{mol}$ relative to IIp, while the transition 


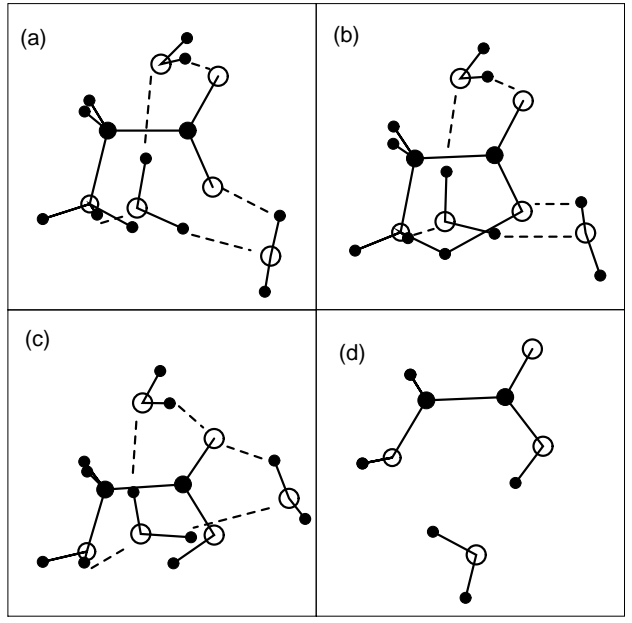

FIG. 7: Structures of a gas phase cluster with one glycine plus 3 water molecules, similar to Ref. 31. (a) IIp; (b) transition state; (c) ZW; (d) bridging water.

state is lowered by $1.5 \mathrm{kcal} / \mathrm{mol}$, similar to the predictions of Ref. 31.

\section{B. Choice of exchange correlation functional}

While the BLYP exchange correlation functional used in this work reproduces the water-water pair correlation function well,$\frac{7}{\underline{7}}$ it is known to overestimate correlation effects in hydrogen bonded systems and slightly underestimate proton transfer energy barriers. For example, from a survey of proton transfer barriers in molecular systems $, 69,70,71,72$ B3LYP and MP2 results are found to be within $0.6 \mathrm{kcal} / \mathrm{mol}$ with the highest level of quantum chemistry calculations (QCISD $(\mathrm{T})$ and $\operatorname{CCSD}(\mathrm{T})$ ) in a suite of test cases, while the BLYP $\Delta E^{*}$ is at most $2.3 \mathrm{kcal} / \mathrm{mol}$ less than B3LYP predictions. This suggests that the accuracy of the small, IIp $\rightarrow \mathrm{ZW}$ proton transfer barrier predicted using the BLYP functional can be assessed by comparing with B3LYP results in a suitable basis set.

Again we consider a supermolecule geometry similar to that in Ref. 31, using the same basis and refined basis sets as before The B3LYP functional predicts the aqueous phase IIp $\rightarrow \mathrm{ZW} \Delta G$ and $\Delta G^{*}$ are predicted to be $-4.0 \mathrm{kcal} / \mathrm{mol}$ and $2.6 \mathrm{kcal} / \mathrm{mol}$, respectively. These predictions are similar to Kassab et al.'s $\Delta G=-5.4 \mathrm{kcal} / \mathrm{mol}$ and $\Delta G^{*}=2.2 \mathrm{kcal} / \mathrm{mol}$, although those were obtained using a smaller basis set.

The BLYP functional, which we use in our AIMD simulations, predicts that the aqueous phase IIp $\rightarrow \mathrm{ZW} \Delta G$ and $\Delta G^{*}$ are $-5.4 \mathrm{kcal} / \mathrm{mol}$ and $1.2 \mathrm{kcal} / \mathrm{mol}$, respec- tively. This confirms that calculations with the BLYP functional yield similar but slightly smaller $\Delta G^{*}$ 's than with the B3LYP functional for the proton transfer systems of interest. The more reliable B3LYP model will raise $\Delta G^{*}$ slightly, but this is opposite in sign to the $\mathrm{ZPE}$ correction and the two partially cancel. The BLYP and B3LYP $\Delta G$ 's also differ by only $1 \mathrm{kcal} / \mathrm{mol}$. Yet the $\Delta G$ of $-5.4 \mathrm{kcal} / \mathrm{mol}$ predicted using the BLYP functional with a supermolecule plus dielectric continuum approach is considerably smaller than the AIMD umbrella sampling prediction of $-11.2 \mathrm{kcal} / \mathrm{mol}$, even after the latter is corrected for ZPE.

Thus there is a several $\mathrm{kcal} / \mathrm{mol}$ discrepancy between our BLYP-based AIMD results, and what we compute using BLYP and a static 3-water supermolecule plus dielectric continuum calculation. We tentatively assign this to the fact that 3 water molecules are not sufficient to model the $-\mathrm{NH}_{3}^{+}$and $-\mathrm{COO}^{-}$first hydration shells, although the supermolecule predictions also show some dependence on basis set and choice of dielectric continuum model. The limitations of using a small number of water molecules have been pointed out already in the literature ${ }^{73.74}$

\section{Appendix B: Electrostatic boundary conditions and finite size effects in AIMD simulations}

In this section we describe the finite size corrections to the hydration free energy $\Delta G_{\text {hyd }}$ of a glycine zwitterion in water, which is closely related to the potential of mean force $\Delta G(R)$ associated with the intramolecular proton transfer in glycine. We consider two estimates of this correction: that due to a dielectric continuum argument, and explicit calculations of the AIMD potential of mean force profile by varying the simulation box size. Both indicate that the $11.8 \AA$ simulation box is adequate for glycine zwitterion in water.

The following arguments are general and illustrate the ability of $a b$ initio molecular dynamics (AIMD) methods to successfully predict the hydration free energies of dipolar species in water.

\section{Dielectric Continuum Estimate}

The effect of periodic boundary conditions on liquid state computer simulations has been a well-studied subject ${ }^{75}$ In our case, zwitterion glycine has a relatively large dipole moment; we compute a value of order 15 Debye in the gas phase. Nevertheless, using a dielectric continuum estimate, we will show that the relatively small box size has little effect on the glycine zwitterion $\Delta G_{\text {hyd }}$.

Ewald sums and periodic boundary conditions are almost invariably used in AIMD simulations, including those reported in this work. Consider the $\Delta G_{\text {hyd }}$ of a sin- 


\begin{tabular}{|l|l|l|l|l|}
\hline$\triangleright$ & $\triangleright$ & $\triangleright$ & $\triangleright$ & $\triangleright$ \\
\hline$\triangleright$ & $\triangleright$ & $\triangleright$ & $\triangleright$ & $\triangleright$ \\
\hline$\triangleright$ & $\triangleright$ & $\triangleright$ & $\triangleright$ & $\triangleright$ \\
\hline$\triangleright$ & $\triangleright$ & $\triangleright$ & $\triangleright$ & $\triangleright$ \\
\hline$\triangleright$ & $\triangleright$ & $\triangleright$ & $\triangleright$ & $\triangleright$ \\
\hline
\end{tabular}

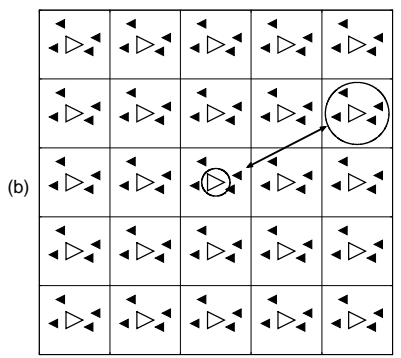

FIG. 8: Illustrations of dielectric continuum estimates for a dipolar solute embedded in a high dielectric solvent. Hollow triangle: solute; filled triangle: solvent molecules. The triangles point in the directions of the dipole moments. The black circles illustrate which interactions between the solute and the periodic images are accounted for. (a) solute-solute image interaction only (attractive, corrected by Eq. 2); (b) all interactions (repulsive, corrected by Eq. 3); (b) only solutesolvent image (repulsive, corrected by Eq. 4). With pair-wise additive force fields, (a) and (c) add up to (b); thus they cancel to a large extent in a medium with a high dielectric constant. Despite the particulate representation of solvent depicted herein, for the purpose of this discussion, the solvent is treated as a dielectric continuum.

gle dipolar solute (molecule) at infinite dilution computed using Ewald summation. In a vacuum (Fig. 8a), the solute-solute image contribution to the computed configurational energy is given by ${ }^{76}$

$$
E_{\text {solute-solute }}=-\left(2 \pi \mathbf{d}^{2} / 3 L^{3}\right)
$$

in atomic units, where $\mathbf{d}$ is the dipole moment and $L$ is length of the cubic simulation cell. This solute-solute image interaction due to the Ewald sum is attractive in a cubic box; the boundary conditions overestimate the magnitude of $\Delta G_{\text {hyd }}$. The correct energy of a dipolar solute in vacuum is then the value computed using Ewald sum minus $E_{\text {solute-solute }}$.

For a box size of $L=11.8 \AA$ and $|\mathbf{d}|=15$ Debye, Eq. 2 implies a $\sim+5 \mathrm{kcal} / \mathrm{mol}$ correction in vacuum.

Our zwitterion glycine molecule, however, resides in water, not in vacuum (Fig. 8b). In this case, the finite size contribution $E_{\text {dipole }}$ in Eq. 2 is replaced by

$$
E_{\text {screened }}=-\left(2 \pi \mathbf{d}^{2} / 3 L^{3}\right) / \epsilon_{o}
$$

where $\epsilon_{o}$ is the (relative) static dielectric constant of water. Therefore, the correct result is the Ewald sum value plus $\left|E_{\text {screened }}\right|$. A similar dielectric continuum estimate of screened solute-solute image interaction has been used successfully to understand the finite size effect in the potential of mean force of sodium chloride ion pair separation, computed using force field based molecular dynamics ${ }^{77}$ In that case, as the sodium and chloride ions are pulled apart from their contact ion-pair configuration, a large dipole moment is incurred. Nevertheless, due to the strong aqueous dielectric screening, the use of Ewald sum and periodic boundary conditions still lead to results well converged with box size (see Figs. $1 \& 2$ of Ref. 77.) Estimates similar to Eq. 3 are also used in solid state density functional theory calculations of defect energetics $\underline{76}$

Assuming AIMD water exhibits $\epsilon_{o} \sim 80$, the dielectric continuum estimate of Eq. [3] suggests that the Ewald sum we use entails a correction of less than $+0.1 \mathrm{kcal} / \mathrm{mol}$ to $\Delta G_{\text {hyd }}$ for our box size of $L=11.8 \AA$.

In hydration free energy calculations that employ classical force fields, the corrections due to Ewald sums and periodic boundary conditions are often described from a different perspective ${ }^{78}$ Unlike the case with density functional theory, where the interaction is inherently manybody and not pairwise decomposible, classical force fields typically allow the computation of strictly solute-water interactions. Thus the interaction of a dipolar solute with all periodic images, Fig. 8b, can be unambiguously separated into solute-solute image (Fig. 8a) and solute-water image (Fig. 8c) contributions.

Using explicit force field-based molecular dynamics simulations, Hummer, Pratt, and Garcia ${ }^{78}$ have eloquently discussed the ramification of this separation. If only the solute-water terms is used, the long range solutewater image interaction, pictorially depicted in Fig. 8c, can lead to considerable finite size dependence in $\Delta G_{\text {hyd }}$. If the entire expression of Fig. 8b is used, i.e., if solutesolute self-energy term (Fig. 8a) is also included, finite size dependences become negligable $\stackrel{78}{ }$ From such an analysis, it can be deduced that the solute-water image interaction is repulsive:

$$
E_{\text {solute-water }} \approx+\left(2 \pi \mathbf{d}^{2} / 3 L^{3}\right),
$$

Equation 4 decreases $\Delta G_{\text {hyd }}$. It is equal and opposite to Eq. 2. the self-energy Makov and Payne corrected for a dipole in vacuum, as it should be; compare the expressions in Eq. 19, Ref. 78, with the last term of Eq. 6 in Ref. 76.

The physics behind the success of the solute-solute image self-term in removing finite size effects from the solute-water image interaction (Fig. 8c) is as follows. 
The solute with dipole moment $\mathbf{d}$ causes water dipole moments in its vicinity to align against it. Since water has a large dielectric constant, to a zeroth approximation the screening of the solute dipole moment is complete, and water molecules proximal to the solute exert $\mathrm{a} \sim \mathbf{- d}$ dipole moment per simulation cell. (On average, the simulation cell should have a net dipole moment that approaches zero. For an anology, consider a metal, which has an infinite dielectric constant and cannot support internal electric fields; in that case, it is obvious each unit cell has a zero dipole moment, as long as localized Wannier functions are judiciously used to demarcate the centers of charge.) Thus the solute-water images in the periodically replicated system give a repulsive energy of $+\left(2 \pi \mathbf{d}^{2} / 3 L^{3}\right)$ (Fig. $8 \mathrm{~b}$ ), equal and opposite to the solutesolute image interaction.

The solute-solute image and the solute-water image interactions are not pairwise additive in AIMD simulations, where energies are inherently many-body in nature. Instead, AIMD propagates Newton's equations of motion according to the total energy and forces computed using Ewald summation, which automatically include both long-range solute-solute image (Fig. 8a) and solute-water image interactions (Fig. 8c) . Thus AIMD simulations do not suffer from the large finite size correction associated with Eq. 4. namely the unfavorable solute-water image interaction (Fig. 8c) - as long as $\epsilon_{o}$ is large and the dielectric continuum approximation is valid.

Since water does not have an infinite dielectric constant, the screening of the solute dipole is incomplete, and the solute-water image repulsion (Fig. 8c) should be slightly less than the solute-solute image attraction (Fig. 8a). Equation 3 is an estimate of this residual effect. In the $\epsilon_{o} \rightarrow \infty$ limit, the system is metallic and this correction vanishes. When $\epsilon_{o}=1$, the system is in a vacuum, and Eq. 3. correctly reduces to Eq.2. For $\epsilon_{o} \approx 2$, the above arguments may not be valid because they rely on the assumption of strong dielectric screening.

In summary, our dielectric continuum estimate indicates that the finite size correction for our AIMD glycine zwitterion hydration free energy is less than $0.1 \mathrm{kcal} / \mathrm{mol}$.

\section{Explicit Simulation Results}

The above analysis assumes a dielectric continuum description of water. The particulate nature of water is not taken into account; with a relatively small simulation cell size and a small number of water molecules, it may not be completely valid. To investigate this, we have explicitly varied the simulation cell size in AIMD calculations of the glycine intramolecular proton transfer potential of mean force $(\Delta G(R))$ in two umbrella sampling windows. We use a simulation cell roughly twice the volume used in the main text, containing 98 instead of 52 water molecules. 10 ps AIMD trajectories are used for collecting statistics.

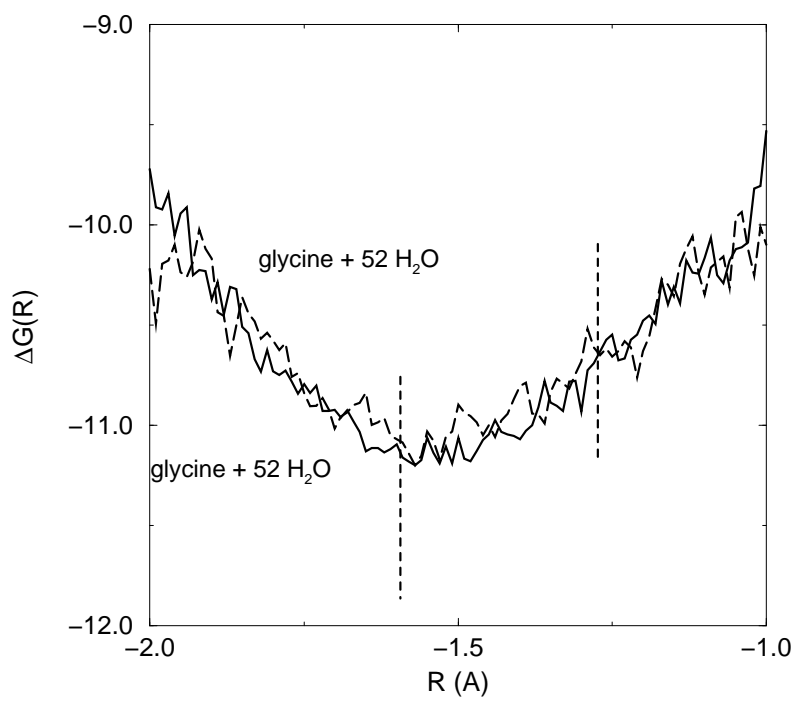

FIG. 9: Finite size effect in $\Delta G(R)$ for glycine in sampling window (f). The blue lines demarcate the $R$ range used in this window for the overall potential of mean force. This is the unconstrained zwitterion window; a wide range of $R$ values are sampled, and the statistics are not as good as in other windows.

First we consider the unconstrained zwitterion sampling window (a). See Fig. 9. This sampling window does not use constraints, and so the glycine zwitterion conformations fluctuate more than in other windows. As a result, the statistics for $\Delta G(R)$ tend to be worse than for other windows. Nevertheless, it can be seen from Fig. 9 that $\Delta G(R)$ predicted for the two simulation cell sizes are within statistical uncertainties of each other within a range of $R$ where $\Delta G(R)$ does not vary by more than 3 $\mathrm{k}_{\mathrm{B}} \mathrm{T}$. The mean hydration structures are similar in both trajectories as well. The average hydration numbers of the larger and smaller simulation cells are with 0.1 water molecules of each other, with the former having $20 \%$ more intramolecular hydrogen bond - well within the statistical uncertainty.

Since the $\Delta G(R)$ profile in window (a) is relatively flat, we also look at window (d). This window contributes half ( $\sim 5 \mathrm{kcal} / \mathrm{mol}$ ) of the overall $\Delta G(R)$, and should shed more light on finite size effects. In Fig. 10, we see that the larger and smaller simulation cells yield $\Delta \Delta G$ of 4.5 and $5 \mathrm{kcal} / \mathrm{mol}$, respectively. The difference is within the statistical uncertainty. From these explicit simulation results, we conclude that the finite size effect should be within our statistical uncertainty. 


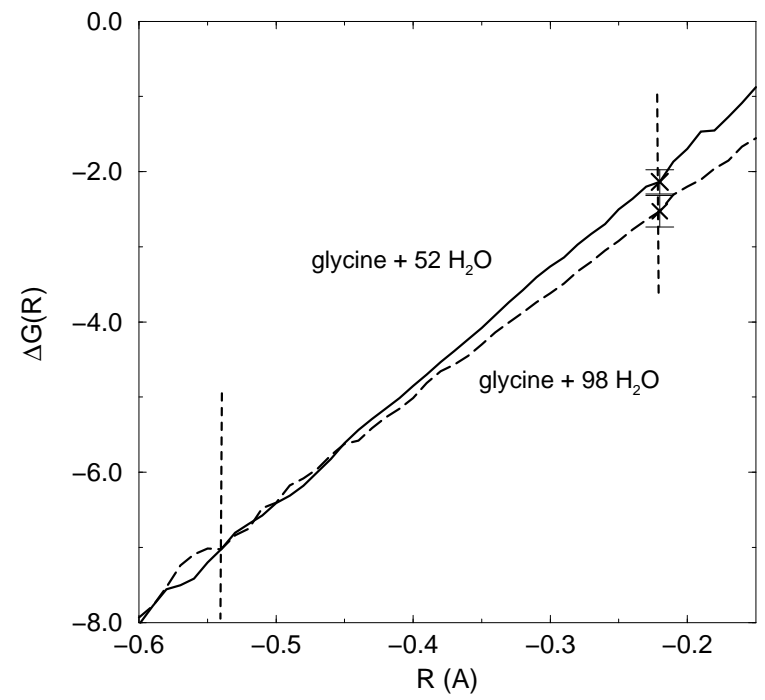

FIG. 10: Finite size effect in $\Delta G(R)$ for glycine in sampling window (d). The blue lines demarcate the $R$ range used in this window for the overall potential of mean force.

1 K.-D. Kreuer, S. J. Paddison, E. Spohr, and M. Schuster, Chem. Rev. 104, 4637 (2004).

2 T. E. Decoursey, Physiol. Rev. 83475 (2003).

${ }^{3}$ I. Andersson and T. C. Taylor, Archives of Biochem. Biophys. 414, 130 (2003).

4 See, for example, C. M. Nimigean, J. S. Chappie, and C. Miller, Biochem. 42, 9263 (2003).

${ }^{5}$ L. G. Cuello, J. G. Romero, D. M. Cortes, and E. Perozo, Biochem. 37, 3229 (1998).

6 See, for example, M. Sprik, J. Phys. Condens. Matter 12, A161 (2000).

7 I-F. W. Kuo, C. J. Mundy, M. J. McGrath, J. I. Siepmann, J. VandeVondele, M. Sprik, J. Hutter, B. Chen, M. L. Klein, F. Mohamed, M. Krack, and M. Parrinello, J. Phys. Chem. B 108, 12990 (2004).

8 I. Ivanov and M. L. Klein, J. Am. Chem. Soc. 124, 13380 (2002).

9 M. Sprik, J. Phys. Condens. Matter 12, A161 (2000); J. E. Davis, N. L. Doltsinis, A. J. Kirby, C. D. Roussev, and M. Sprik, J. Am. Chem. Soc. 124, 6594 (2002).

10 P. L. Geissler, C. Dellago, D. Chandler, J. Hutter, and M. Parrinello, Science 291, 2121 (2001).

11 J. Schlitter and M. Klahn, J. Chem. Phys. 118, 2057 (2003).

12 L. R. Pratt and R. A. LaViolette, Mol. Phys. 94, 909 (1998).

13 Theoretically, it has been shown that at least 3 water molecules are needed to stablize $\mathrm{ZW}$ over $\mathrm{NF}$ in the gas phase: see J. H. Jensen and M. S. Gordon, J. Am. Chem. Soc. 117, 8159 (1995).
14 H. M. Senn, P. M. Margl, R. Schmid, T. Ziegler, and P. E. Blochl, J. Chem. Phys. 118, 1089 (2003); dynamics of the glycine molecule are explicitly treated here.

15 P. Bandyopadhyay, M. S. Gordon, B. Mennucci, and J. Tomasi, J. Chem. Phys. 116, 5023 (2002). A glycine$\left(\mathrm{H}_{2} \mathrm{O}\right)_{8}$ cluster without dielectric continuum correction is also considered in this work.

16 M. Sheinblatt and H. S. Cutowsky, J. Am. Chem. Soc. 86, 4814 (1964).

17 G. Wada, E. Tamura, M. Okina, and M. Nakamura, Bull. Chem. Soc. Jpn 55, 3064 (1982).

18 K. C. Chang and E. Grunwald, J. Phys. Chem. 80, 1422 (1976).

19 E. Grunwald, K. C. Chang, P. L. Skipper, and V. K. Anderson, J. Phys. Chem. 80, 1425 (1976)

20 M. A. Slifkin and S. M. Ali, J. Mol. Liquids 28, 215 (1984).

${ }^{21}$ P. Haberfield, J. Chem. Ed. 57, 346 (1980).

22 Y. Kameda, H. Ebata, T. Usuki, O. Uemura, and M. Misawa, Bull. Chem. Soc. Jpn. 67, 3159 (1994).

23 The designations follow those of A. Császár, J. Am. Chem. Soc. 114, 9568 (1992).

24 Y. Ding and K. Krogh-Jespersen, Chem. Phys. Lett. 199, 261 (1992).

25 S. Xu, J. M. Nilles, K. H. Bowen, Jr., J. Chem. Phys. 119 10696 (2003).

26 See A. G. Császár and A. Perczel, Prog. Biophysics and Mol. Biol. 71, 243 (1999) for a review of amino acid conformers.

27 S. Sirois, E. I. Proynov, D. T. Nguyen, and D. R. Salahub, J. Chem. Phys. 107, 6770 (1997), and references therein. 
28 N. Okuyama-Yoshida, M. Nagaoka, and T. Yamabe, J. Phys. Chem. A 102, 285 (1998).

29 M. Nagaoka, N. Okuyama-Yoshida, and T. Yamabe, J. Phys. Chem. A 102, 8202 (1998).

${ }^{30}$ I. Tuñón, E. Silla, and M. F. Ruiz-López, Chem. Phys. Lett. 321, 433 (2000).

31 E. Kassab, J. Langlet, E. Evleth, and Y. Akacem, Theochem 531, 267 (2000).

32 M. E. Tuckerman, D. Marx, and M. Parrinello, Nature 417, 925 (2002).

${ }^{33}$ W. L. Jorgensen and J.-L. Gao, J. Phys. Chem. 90, 2174 (1986).

34 F. M. L. G. Stamato and J. M. Goodfellow, Int. J. Quant. Chem.: Quant. Biol. Symposium 13, 277 (1986), and references therein.

35 G. Alagona, C. Ghio, and P. A. Kollman, Theochem 166, 385 (1988).

36 G. Alagona, C. Ghio, and P. A. Kollman, J. Am. Chem. Soc. 108, 185 (1986).

37 Computer simulations of aquous amino acid zwitterions and related species include: U. C. Singh, F. K. Brown, P. A. Bash, and P. A. Kollman, J. Am. Chem. Soc. 109, 1607 (1987); P. E. Smith, L. X. Dang, and B. M. Pettitt, J. Am. Chem. Soc. 113, 67 (1991); P. I. Nagy and K. Takács-Novák, J. Am. Chem. Soc. 119, 4999 (1997); D. J. Price, J. D. Roberts, and W. L. Jorgensen, J. Am. Chem. Soc. 120, 9672 (1998); and G. E. Marlow and B. M. Pettitt, J. Am. Chem. Soc. 68, 192 (2003).

38 J. Gao, J. Am. Chem. Soc. 117, 8600 (1995).

39 Y. Kameda, T. Mori, T. Nishiyama, T. Usuki, and O. Uemura, Bull. Chem. Soc. Jpn. 69, 1495 (1996).

40 Y. Kameda, K. Fukuhara, K. Mochiduki, H. Naganuma, T. Usuki, and O. Uemura, J. Non. Cryst. Solids 312-314, 433 (2002).

41 E. Cubero, F. J. Luque, M. Orozco, and J.-L. Gao, J. Phys. Chem. B 107, 1664 (2003).

42 (a) Warshel and Levitt, J. Mol. Biol. 103, 227 (1976); (b) M. J. Field, P. A. Bash, and M. Karplus, J. Comput. Chem. 11, 700 (1990).

43 K. Leung and S. B. Rempe, J. Am. Soc. Chem. 126, 344 (2004).

44 T. Shoeib, G. D. Ruggiero, K. W. M. Siu, A. C. Hopkinson, and I. H. William, J. Chem. Phys. 117, 2762 (2002).

45 A. Fernández-Ramos, Z. Smedarchina, W. Siebrand, and M. Z. Zgierski, J. Chem. Phys. 113, 9714 (2000).

${ }^{46}$ L. R. Pratt and S. B. Rempe, in L. R. Pratt and G. Hummer (Eds.), Simulation and Theory of Electrostatic Interations in Solution (ALP, New York, 1999), pp. 172-201.

47 S. B. Rempe and L. R. Pratt, Fluid Phase Equil. 183-184, $121(2001)$

48 S. B. Rempe, L. R. Pratt, G. Hummer, J. D. Kress, R. L. Martin, and A. Redondo, J. Am. Soc. Chem. 122, 966 (2000)

49 D. Asthagiri, L. R. Pratt, M. E. Paulaitis, and S. B. Rempe, J. Am. Soc. Chem. 126, 1285 (2004)

50 S. B. Rempe, D. Asthagiri, and L. R. Pratt, Phys. Chem. Chem. Phys. 6, 1966 (2004)

51 CPMD Version 3.4, J. Hütter, P. Ballone, M. Bernasconi, P. Focher, E. Fois, S. Goedecker, D. Marx, M. Parrinello, and M. Tuckerman, MPI für Festkörperforschung and IBM Research Laboratory (1990-1998), Copyright IBM 1990,
1997, Copyright MPI 1997.

52 A. D. Becke, Phys. Rev. A 38, 3098 (1988).

53 C. T. Lee, W. T. Yang, and R. G. Parr, Phys. Rev. B 37, 785 (1988).

54 N. Troullier and J. L. Martins, Phys. Rev. B 43, 1993 (1991).

55 F. Brugé, M. Bernasconi, and M. Parrinello, J. Am. Soc. Chem. 121, 10883 (1999), and references therein.

${ }^{56}$ T. H. Lilley (G. C. Barret, Ed.) The Chemistry and Biochemistry of Amino Acids, (Chapman \& Hall, 1985).

57 Our fixed volume predictions actually correspond to the Helmholtz free energy. Nevertheless, we will use " $\Delta G$ " throughout, since that appears to be the common usage in this field.

58 See, for example, D. Chandler, Introduction to Modern Statistical Mechanics (Oxford University Press, New York, 1987).

59 As discussed in the text, rotation about the $\mathrm{N}-\mathrm{C}$ bond renders the three ammonium group protons equivalent. We only observe this rotation, however, when glycine is in the zwitterion form - when the reaction coordinate $R<-2 \AA$. For the other sampling windows, such a rotation cannot occur until $R<-2 \AA$ - i.e., unless the system leaves the pertinent sampling windows, which it is prevented from doing by the umbrella sampling constraints on the glycine geometry (Table 1). So as long as we use an unconstrained $\mathrm{ZW}$ window and constrained sampling windows elsewhere, $\Delta G(R)$ is unambiguous. This is one reason we elect to use umbrella sampling rather than the more common integration of constrained force method. For general ways to deal with deprotonation of atoms with multiple protons attached, see M. Sprik, Chem. Phys. 259, 139 (2000).

60 P. Bolhuis and D. Chandler, J. Chem. Phys. 113, 8154 (2000).

61 See the discussions in Kassab et al. ${ }^{31}$ and Ref. 55 therein.

62 K. Toukan and A. Rahman, Phys. Rev. B 31, 2643 (1985).

63 Our plane-wave based QM/MM capability is built into the plane-wave electronic structure code VASP [G. Kresse and J. Furthmüller, Phys. Rev. B 54, 11169 (1996); Comput. Mater. Sci. 6, 15 (1996)]. The QM/MM algorithm is similar to that of A. Laio, J. VandeVondele, and U. Rothlisberger, J. Chem. Phys. 116, 6941 (2002), except that, given the small box size, the QM and MM regions are coupled together directly in Fourier space.

64 The average hydration number, $\mathrm{N}_{\mathrm{w}}$, obtains by integrating the pair correlation $g(r)$ of the pertinent atom with the water $\mathrm{O}$ or $\mathrm{H}$ atom to its first minimum.

65 Recall that a QM/MM estimate is even larger ${ }^{44}$

66 A. D. Becke, J. Chem. Phys. 98, 5648 (1993).

67 M. J. Frisch et al., Gaussian 98 (Revision A.2), Gaussian Inc., Pittsburgh, PA., 1998.

68 L. R. Pratt, G. J. Tawa, G. Hummer, A. E. Garcia and S. A. Corcelli, Int. J. Quant. Chem. 64, 121 (1997); with the radii defined as in E. V. Stefanovich and T. N. Truong, Chem. Phys. Lett. 244, 65 (1995).

69 V. Barone, L. Orlandini, and C. Adamo, Chem. Phys. Lett. 231, 295 (1994).

70 V. Barone, L. Orlandini, and C. Adamo, Int. J. Quantum Chem. 56, 697 (1995).

71 V. Barone and C. Adamo, J. Chem. Phys. 105, 11007 
(1996).

72 S. Sadhukhan, D. Muñoz, C. Adamo, and G. E. Scuseria, Chem. Phys. Lett. 83, 306 (1999).

73 D. Sicinska, P. Paneth, and D. G. Truhlar, J. Phys. Chem. B 106, 2708 (2002).

${ }^{74}$ Note that an MP2 based, 6 water supermolecule plus dielectric continuum calculation predicts $\Delta G=10.3$ $\mathrm{kcal} / \mathrm{mol}$ after correcting for ZPE ${ }^{45}$ However, this supermolecule has a bridging water molecule between the $-\mathrm{NH}_{3}^{+}$ and $-\mathrm{COO}^{-}$groups, which is not observed in our simula- tions.

75 L. R. Pratt and S. W. Haan, J. Chem. Phys. 74, 1864 (1981); ibid, 74, 1873 (1981).

76 G. Makov and M. C. Payne, Phys. Rev. B 51, 4014 (1995).

77 D. E. Smith and A. D. J. Haymet, Fluid Phase Equil. 88, 79 (1993).

78 G. Hummer, L. R. Pratt, and A. E. Garcia, J. Pnys. Chem. 99, 14188 (1995). 